\title{
Rickettsia tamurae sp. nov., isolated from Amblyomma testudinarium ticks
}

\author{
Correspondence \\ Pierre-Edouard Fournier \\ Pierre-Edouard.Fournier@ \\ medecine.univ-mrs.fr
}

\author{
Pierre-Edouard Fournier, ${ }^{1}$ Nobuhiro Takada, ${ }^{2}$ Hiromi Fujita ${ }^{3}$ \\ and Didier Raoult ${ }^{1}$ \\ ${ }^{1}$ Unité des rickettsies, IFR 48, CNRS UMR 6020, Faculté de médecine, Université de la \\ Méditerranée, 27 Boulevard Jean Moulin, 13385 Marseille cedex 05, France \\ ${ }^{2}$ Department of Pathological Sciences, Faculty of Medical Sciences, University of Fukui, \\ Matsuoka, Fukui 910-1193, Japan \\ ${ }^{3}$ Ohara Research Laboratory, Ohara General Hospital, Fukushima 960-0195, Japan
}

In Japan, Rickettsia japonica (Uchida et al., 1992), causing Japanese spotted fever (Mahara et al., 1985), was the first described spotted fever rickettsia. $R$. japonica has been identified in several tick species including Haemaphysalis longicornis, H. flava, H. formosensis, H. hystricis, Dermacentor taïwanensis and Ixodes ovatus (Fournier et al., 2002). In addition to R. japonica, another species, Rickettsia helvetica, has been isolated from ticks in Japan, being found in Ixodes persulcatus and Ixodes monospinosus (Fournier et al., 2002). Several other rickettsial isolates have been obtained from I. ovatus and Amblyomma testudinarium ticks (Fournier et al., 2002). These include a rickettsial isolate, $\mathrm{AT}-1^{\mathrm{T}}$, cultivated in L929 cells from an A. testudinarium nymph collected in Anan, in the Tokushima prefecture (Fujita et al., 1999). Forty-five further rickettsial isolates were obtained from A. testudinarium ticks collected in Japan (Fujita et al., 1996; Takada et al., 2001) and were demonstrated to be genetically highly similar to AT-1 ${ }^{\mathrm{T}}$ (Fujita et al., 1996; Takada et al., 2001; Fournier et al., 2002). So far, strains conspecific with Rickettsia sp. strain AT- $1^{\mathrm{T}}$ have not been found in any other tick species. Previously, we demonstrated that Rickettsia sp. strain AT- $1^{\mathrm{T}}$ was most closely related to strain IRS4, an uncultivated Slovakian rickettsia from Ixodes ricinus (Sekeyova et al., 2000) that was later shown to be closely related to 'Rickettsia monacensis' (Simser et al., 2002), the name of which has not yet been validly published.

The GenBank/EMBL/DDBJ accession numbers for the $16 \mathrm{~S}$ rRNA, gltA, ompA, ompB and sca4 gene sequences of strain $A T-1^{\top}$ are respectively AY049981, AF394896, DQ103259, DQ113910 and DQ113911.
Preliminary mouse serotyping results and sequence comparison of the 16S rRNA gene ( $r r s)$ and gltA genes suggested that Rickettsia sp. strain AT- $1^{\mathrm{T}}$ exhibits unique serotypic and genotypic characteristics among spotted fever rickettsiae (Takada et al., 2001; Fournier et al., 2002). Herein, using multigene sequencing and mouse serotyping, we evaluated whether this rickettsia fulfils the minimum requirements to be classified within a novel species.

DNA from Rickettsia sp. strain AT- $1^{\mathrm{T}}$ was extracted using the QIAamp tissue kit (Qiagen) according to the manufacturer's instructions. PCR amplification and sequencing of the $5^{\prime}$-end of the ompA gene and the complete ompB and $s c a 4$ genes were attempted using the primers and conditions described previously (Fournier et al., 1998; Roux \& Raoult, 2000; Sekeyova et al., 2001). PCR products were sequenced twice in each direction. $r r s$ and $g l t A$ nucleotide sequences from Rickettsia sp. strain AT- $1^{\mathrm{T}}$, rrs, gltA, ompA, and, when available, sca4 nucleotide sequences from its closest rickettsia relatives, Rickettsia sp. strain IRS4 and ' $R$. monacensis', as well as rrs, gltA, ompB and sca4 nucleotide sequences from the closest Rickettsia species with a validly published name, $R$. helvetica, were retrieved from GenBank. Sequences were edited by removal of primer sequences from the $5^{\prime}$ and $3^{\prime}$ ends. Only pairwise transitions and transversions between sequences, not deletions and insertions, were taken into account to calculate the degree of sequence similarity. Based on ompA sequences, Rickettsia sp. strain $\mathrm{AT}-1^{\mathrm{T}}$ was identical to Rickettsia sp. strain ATT (GenBank accession no. AF483202), detected by PCR in A. testudinarium in Thailand (Hirunkanokpun et al., 2003). Numbers of nucleotide 
substitutions between Rickettsia sp. strain $\mathrm{AT}-1^{\mathrm{T}}$ and $R$. helvetica were 15 (98.9\% nucleotide sequence similarity), $47(96 \cdot 1 \%), 257(94 \cdot 7 \%)$ and $275(90 \cdot 9 \%)$, respectively, for the rrs, gltA, ompB and sca4 genes. Thus, for each of these four genes, Rickettsia sp. strain AT- $1^{\mathrm{T}}$ exhibited similarity rates with $R$. helvetica lower than the cut-offs proposed to classify rickettsial isolates within a species $(99 \cdot 8,99 \cdot 9,99 \cdot 2$ and $99 \cdot 3 \%$, respectively, for the rrs, gltA, ompB and sca 4 genes; Fournier et al., 2003). Therefore, on the basis of genotypic criteria, Rickettsia sp. strain $\mathrm{AT}-1^{\mathrm{T}}$ did not belong to the species $R$. helvetica. In addition, it was also classified into a species distinct from Rickettsia sp. strain IRS4 (Sekeyova et al., 2000), with degrees of sequence similarity between these two rickettsiae being $99 \cdot 3,98 \cdot 7,94 \cdot 0$ and $97 \cdot 8 \%$, respectively, for the $r r s, g l t A, o m p A$ and sca4 genes. Finally, when compared to ' $R$. monacensis' (Simser et al., 2002), Rickettsia sp. strain AT- $1^{\mathrm{T}}$ exhibited degrees of sequence similarity of $98 \cdot 5,99 \cdot 1$ and $93 \cdot 6 \%$, respectively, for the $r r s$, gltA and ompA genes, and thus these two rickettsiae belong to distinct species. A dendrogram inferred from ompB sequences by the neighbour-joining method, showing the position of strain AT- $1^{\mathrm{T}}$ within the genus Rickettsia, is shown in Fig. 1.

Mouse serotyping was conducted as described previously (Philip et al., 1978). We used as antigens Rickettsia sp. strain $\mathrm{AT}-1^{\mathrm{T}}$ and $R$. helvetica $\mathrm{C9P} 9^{\mathrm{T}}$, cultivated on Vero cells (ATCC CRL-1587) as described previously (Marrero \& Raoult, 1989). For each antigen, five BALB/c mice were inoculated intravenously with purified bacterial suspension $\left(0 \cdot 1 \mathrm{ml}\right.$ containing $10^{4}$ bacteria) without adjuvant. On day 7 , mice were boosted with a similar inoculum. On day 10, mice were anaesthetized and exsanguinated by cardiac puncture. Sera from each group of mice were pooled. Microimmunofluorescence (MIF) was performed and the specificity difference (SPD) was calculated as described previously (Philip et al., 1978). If the SPD was $\geqslant 3$, the isolates were assumed to belong to different serotypes. Using serum from mice immunized with Rickettsia sp. strain AT- $1^{\mathrm{T}}$, we found MIF antibody titres of $1: 400$ and $1: 100$ to Rickettsia sp. strain $\mathrm{AT}-1^{\mathrm{T}}$ and $R$. helvetica $\mathrm{C} 9 \mathrm{P} 9^{\mathrm{T}}$, respectively. Using serum from mice immunized with $R$. helvetica $\mathrm{CPP9}^{\mathrm{T}}$, we found MIF antibody titres of $1: 800$ and $1: 100$ to $R$. helvetica $\mathrm{C} 9 \mathrm{P9} 9^{\mathrm{T}}$ and Rickettsia sp. strain $\mathrm{AT}-1^{\mathrm{T}}$, respectively. On the basis of these results, the SPD between the two rickettsiae was 7 . Therefore, the genotypic and serotypic specificity of Rickettsia sp. strain AT- $1^{\mathrm{T}}$ support its classification within a distinct species.

On the basis of genotypic analyses, we have previously proposed the classification of Rickettsia sp. strain AT-1 ${ }^{\mathrm{T}}$ within a novel species (Fournier et al., 2002). Our current results support this proposal. Thus, we formally propose the creation of Rickettsia tamurae sp. nov., containing strain AT $-1^{\mathrm{T}}$ as the type strain. This rickettsia has been found in Japan and Thailand.

Following discussions held at the meetings of the International Committee on Systematics of Prokaryotes (ICSP) and its Judicial Commission (JC) in San Francisco in 2005, and in anticipation of the published minutes of these meetings, a committee consisting of the Chairman of the ICSP, the Chairman of the JC of the ICSP and the Editor of the International Journal of Systematic and Evolutionary Microbiology has granted an exception in this instance to Rule 27(3) of the Bacteriological Code governing the deposit of type material in two different collections in two different countries.

\section{Description of Rickettsia tamurae sp. nov.}

Rickettsia tamurae (ta.mu'rae. N.L. gen. masc. n. tamurae of Tamura, named in honour of the Japanese rickettsiologist

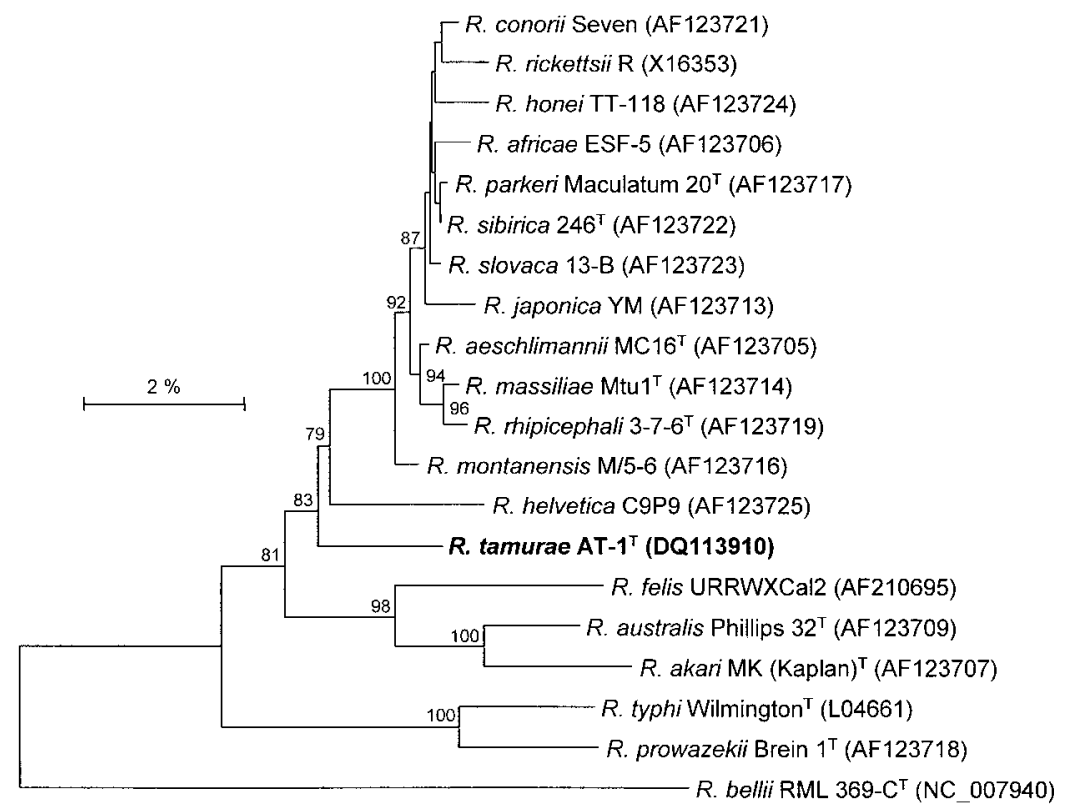

Fig. 1. Unrooted dendrogram showing the phylogenetic position of Rickettsia sp. strain AT $-1^{\top}$ among Rickettsia species with validly published names inferred from comparison of $\mathrm{omp} B$ sequences by the neighbour-joining method. Bootstrap values $>75 \%$ are indicated at nodes. Bar, $2 \%$ nucleotide sequence divergence. 
Dr Akira Tamura, who contributed to our knowledge of rickettsiae and rickettsioses in Japan).

Gram-negative, obligately intracellular bacterium. Grows on Vero cells at $32{ }^{\circ} \mathrm{C}$ in minimal essential medium supplemented with $2 \%$ fetal calf serum and $2 \mathrm{mg}$ L-glutamine $\mathrm{ml}^{-1}$. Non-motile.

The type strain is strain AT- $1^{\mathrm{T}}\left(=\mathrm{CSUR} R 1^{\mathrm{T}}\right)$, which was isolated from an Amblyomma testudinarium tick in 1993 in Tokushima prefecture, Japan (Fujita et al., 1999). The type strain has been deposited in the Collection de souches de l'Unité des Rickettsies (CSUR), World Health Organization Collaborative Center for Rickettsioses, Borrelioses and Tickborne Infections, Marseille, France. Attempts are also being made to deposit the type strain in the American Type Culture Collection.

\section{Acknowledgements}

The part of this work carried out in Japan was supported in part by Grants-in-Aid for Scientific Research from the Japan Society for the Promotion of Science (grant nos 13576005 and 16406008).

\section{References}

Fournier, P. E., Roux, V. \& Raoult, D. (1998). Phylogenetic analysis of spotted fever group rickettsiae by study of the outer surface protein rOmpA. Int J Syst Bacteriol 48, 839-849.

Fournier, P. E., Fujita, H., Takada, N. \& Raoult, D. (2002). Genetic identification of rickettsiae isolated from ticks in Japan. J Clin Microbiol 40, 2176-2181.

Fournier, P. E., Dumler, J. S., Greub, G., Zhang, J., Wu, Y. \& Raoult, D. (2003). Gene sequence-based criteria for identification of new rickettsia isolates and description of Rickettsia heilongjiangensis sp. nov. J Clin Microbiol 41, 5456-5465.
Fujita, H., Takada, N. \& Tsuboi, Y. (1996). Survey of ixodid ticks (Acarina: Ixodidae) and tick-borne spotted fever group rickettsiae in Tokunoshima Island, Japan. Med Entomol Zool 47, 15-21.

Fujita, H., Watanabe, Y., Ishikura, M. \& Takada, N. (1999). List of all isolates of spotted fever group rickettsiae from ticks in Japan 1993-1998. Ann Rep Ohara Hosp 42, 45-50.

Hirunkanokpun, S., Kittayapong, P., Cornet, J. P. \& Gonzalez, J. P. (2003). Molecular evidence for novel tick-associated spotted fever group rickettsiae from Thailand. J Med Entomol 40, 230-237.

Mahara, F., Koga, K., Sawada, S. \& 8 other authors (1985). The first report of the rickettsial infections of spotted fever group in Japan; three clinical cases. Jpn J Assoc Infect Dis 59, 1165-1172.

Marrero, M. \& Raoult, D. (1989). Centrifugation-shell vial technique for rapid detection of Mediterranean spotted fever rickettsia in blood culture. Am J Trop Med Hyg 40, 197-199.

Philip, R. N., Casper, E. A., Burgdorfer, W., Gerloff, R. K., Hugues, L. E. \& Bell, E. J. (1978). Serologic typing of rickettsiae of the spotted fever group by microimmunofluorescence. J Immunol 121, 1961-1968.

Roux, V. \& Raoult, D. (2000). Phylogenetic analysis of members of the genus Rickettsia using the gene encoding the outer-membrane protein rOmpB (ompB). Int J Syst Evol Microbiol 50, 1449-1455.

Sekeyova, Z., Fournier, P. E., Rehacek, J. \& Raoult, D. (2000). Characterization of a new spotted fever group rickettsia detected in Ixodes ricinus (Acari: Ixodidae) collected in Slovakia. J Med Entomol 37, 707-713.

Sekeyova, Z., Roux, V. \& Raoult, D. (2001). Phylogeny of Rickettsia spp. inferred by comparing sequences of 'gene D', which encodes an intracytoplasmic protein. Int J Syst Evol Microbiol 51, 1353-1360.

Simser, J. A., Palmer, A. T., Fingerle, V., Wilske, B., Kurtti, T. J. \& Munderloh, U. G. (2002). Rickettsia monacensis sp. nov., a spotted fever group rickettsia, from ticks (Ixodes ricinus) collected in a European city park. Appl Environ Microbiol 68, 4559-4566.

Takada, N., Fujita, H., Yano, Y., Ishiguro, F., Iwasaki, H. \& Masuzawa, T. (2001). First records of tick-borne pathogens, Borrelia, and spotted fever group rickettsiae in Okinawajima Island, Japan. Microbiol Immunol 45, 163-165.

Uchida, T., Uchiyama, T., Kumano, K. \& Walker, D. H. (1992). Rickettsia japonica sp. nov., the etiological agent of spotted fever group rickettsiosis in Japan. Int J Syst Bacteriol 42, 303-305. 